(1)

CrossMark

\title{
What is a COPD exacerbation? Current definitions, pitfalls, challenges and opportunities for improvement
}

\author{
Victor Kim (10 ${ }^{1}$ and Shawn D. Aaron $\mathbb{1}^{2}$ \\ Number 1 in the series "Controversies in COPD: What Can be Done to Move the \\ Field Forward?" \\ Edited by D.D. Sin
}

\begin{abstract}
Affiliations: ${ }^{1}$ Lewis Katz School of Medicine at Temple University, Philadelphia, PA, USA. ${ }^{2}$ The Ottawa Hospital Research Institute, University of Ottawa, Ottawa, ON, Canada.

Correspondence: Shawn D. Aaron, The Ottawa Hospital Research Institute, General Campus, 501 Smyth Road, Ottawa, Ontario, Canada, K1H 8L6. Email: saarondohri.ca
\end{abstract}

@ERSpublications

Current state of the art in COPD exacerbations, with respect to definitions, associated pitfalls and challenges, and opportunities for improvement http://ow.ly/7ru430lUFJ1

Cite this article as: Kim V, Aaron SD. What is a COPD exacerbation? Current definitions, pitfalls, challenges and opportunities for improvement. Eur Respir J 2018; 52: 1801261 [https://doi.org/10.1183/ 13993003.01261-2018].

ABSTRACT Chronic obstructive pulmonary disease (COPD) is a chronic illness that can be periodically punctuated by exacerbations, characterised by acute worsening of symptoms, including increased dyspnoea, cough, sputum production and sputum purulence. COPD exacerbations are common and have important clinical and economic consequences, including lost work productivity, increased utilisation of healthcare resources, temporary or permanent reductions in lung function and exercise capacity, hospitalisation, and sometimes death. Over the past two decades, clinicians and researchers have broadened their treatment goals for COPD to extend beyond improving lung function and symptoms, and have begun to address the importance of preventing and reducing exacerbations. However, despite the best efforts of clinicians and guideline committees, current definitions of COPD exacerbations are imperfect and fraught with problems. The cardinal symptoms of a COPD exacerbation are nonspecific and can result from acute cardiorespiratory illnesses other than COPD. A proposed definition, which may be more specific than current definitions, suggests that COPD exacerbation be defined as an acute or subacute worsening of dyspnoea ( $\geqslant 5$ on a visual analogue scale that ranges from 0 to 10) sometimes but not necessarily accompanied by increased cough, sputum volume and/or sputum purulence. Necessary laboratory criteria for an exacerbation include oxygen desaturation $\leqslant 4 \%$ below that of stable state, elevated levels of circulating blood neutrophils or eosinophils ( $\geqslant 9000$ neutrophils $\cdot \mathrm{mm}^{-3}$ or $\geqslant 2 \%$ blood eosinophils) and elevated C-reactive protein $\left(\geqslant 3 \mathrm{mg} \cdot \mathrm{L}^{-1}\right)$, without evidence of pneumonia or pulmonary oedema on chest radiography and with negative laboratory test results for other aetiologies. Herein, we discuss the current state of the art with respect to how we define COPD exacerbations, associated pitfalls and challenges, and opportunities for improvement. 


\section{Introduction}

Chronic obstructive pulmonary disease (COPD) is a chronic illness that can be periodically punctuated by acute worsening of symptoms characterised clinically by increased dyspnoea, cough, sputum production and sputum purulence. This acute worsening of symptoms has been termed acute exacerbation of COPD (AECOPD). COPD exacerbations are common and have important clinical and economic consequences, including lost work productivity, acute decline in quality of life, temporary or permanent reduction in lung function and exercise capacity, hospitalisation, and sometimes death [1-7]. Prospective studies have shown that the most important risk factor for future exacerbations is a patient history of prior exacerbations [8]. Other risk factors for exacerbations include low baseline lung function, increased symptom burden, radiographic evidence of emphysema and a history of chronic bronchitis [8-10].

Over the past two decades, clinicians and researchers have broadened their treatment goals for chronic COPD to extend beyond improving lung function and symptoms, and have begun to address the importance of maintenance treatment of stable COPD to prevent or decrease the incidence of exacerbations. Many randomised clinical trials of chronic COPD therapies now use AECOPD event rates as their primary study outcome to judge the efficacy of chronic COPD therapies [11, 12]. Given the emerging clinical, economic and research importance of AECOPD, it is critical that COPD exacerbations are rigorously defined and that exacerbation events are easily ascertained and quantified both in clinical practice and in research studies.

It is important that we gain a better understanding of what an exacerbation is, so that we can improve upon the currently accepted definitions and clarify these common and sometimes life-threatening events. The objective of this narrative review is to discuss the current state of the art with respect to how we define COPD exacerbations, associated pitfalls and challenges, and opportunities for improvement. Relevant articles for this review were retrieved from the Medline and PubMed databases using the following search terms: "COPD exacerbation", "exacerbations of COPD" and "COPD exacerbation definition". Abstracts were assessed for potential relevance to the topic and applicable articles were included in this review.

\section{Current definition}

Exacerbations can be defined using symptom-based or event-based definitions, or a combination of the two. Symptom-based definitions rely on patient-reported worsening of respiratory symptoms either to a healthcare practitioner or within a symptom diary. Typical symptoms of AECOPD include increased dyspnoea, cough, sputum volume and sputum purulence. In contrast, event-based definitions capture patients whose conditions have changed enough to require a change in treatment.

The first definition of COPD exacerbation dates to the 1980s and was a symptom-based definition focused exclusively on three cardinal symptoms, i.e. the "increase or onset of shortness of breath, sputum production and/or sputum purulence" [13]. Later an event-driven definition was introduced, and exacerbations were defined as "a worsening of COPD symptoms requiring changes to normal treatment, including antimicrobial therapy, short courses of oral steroids and other bronchodilator therapy" [14]. A subsequent definition proposed by a consensus conference in 2000 defined an exacerbation as "an acute event characterised by a worsening of the patient's respiratory symptoms that is beyond normal day-to-day variations and necessitates a change in regular medication, in a patient with underlying COPD" [15]. The 2018 Global Initiative for Chronic Obstructive Lung Disease (GOLD) definition of AECOPD uses an event-based definition. The 2018 GOLD document defines COPD exacerbation as "an acute worsening of respiratory symptoms that results in additional therapy". Exacerbations are classified as 1) mild if they are treated with short-acting bronchodilators only; 2) moderate if they are treated with short-acting bronchodilators plus antibiotics and/or oral corticosteroids; or 3) severe if the patient visits the emergency room or requires hospitalisation because of an exacerbation [16].

The 2018 GOLD definition integrates the concept of clinical worsening of an acute event with a change in the regular treatment for COPD. The definition includes a compromise between symptom- and event-driven approaches adopted by earlier definitions. It provides a grading system from mild to severe based on management and therapy. Although not stated within the definition, a vague description of symptoms ("an acute worsening of respiratory symptoms") should prompt consideration of a differential diagnosis for acute events that can mimic an AECOPD. These events can be pulmonary (e.g. pneumonia, pulmonary thromboembolism) or non-pulmonary in nature (e.g. congestive heart failure, acute coronary syndromes or anaemia) [17].

\section{Advantages and disadvantages of symptom-based definitions of AECOPD}

An obvious advantage of using a symptom-based definition is that symptoms are important and are the primary concern of the patient. Therefore, defining an exacerbation based on symptoms has clinical 
relevance for patients and caregivers. A further advantage is that validated tools to capture symptom-based AECOPDs exist and include patient diary cards [18] and the validated Exacerbation of Chronic Pulmonary Disease Tool (EXACT) [19].

The disadvantages include the subjective nature of symptom-based definitions. In some cases, it is difficult for the patient and the clinician to decide if a patient's symptoms are "increased" more than usual [19]. In addition, numerous studies have shown that symptom-based AECOPDs often go unreported, leading to an under-estimation of patient exacerbation rates [20,21]. Finally, paper-based diary cards and home-based symptom assessment tools are plagued by poor adherence and recall biases related to delays in entering symptoms (diary hoarding), thus leading to retrospective record entry and reduced data accuracy [22]. Use of electronic diaries, rather than paper-based methods, may allow for daily prompting of patients and help to alleviate delays in data entry [22].

Studies of the EXACT confirmed that it reliably assesses symptom severity and that EXACT scores are significantly elevated at exacerbation compared to baseline, stable-state values $[19,23]$. Independent studies have also confirmed that EXACT scores increase at COPD exacerbation, and the magnitude of the increase reflects the severity of the event in terms of treatment, systemic inflammation, airflow limitation and symptom recovery time [24]. However, studies comparing the EXACT to daily patient diary cards and physician assessment have shown marked disparity in exacerbation assessments. Over a 2-year period, only $28 \%$ of daily diary card exacerbations were picked up by the EXACT questionnaire, indicating poor reliability between standard tools used to identify symptom-based exacerbations [24].

\section{Advantages and disadvantages of event-based definitions of AECOPD}

Event-based definitions circumvent the problems associated with identifying a group of symptoms by capturing patients whose conditions have changed enough to require a change in treatment or hospitalisation. An advantage of event-based definitions is that they capture exacerbations that are more clinically important than purely symptom-based exacerbations. Event-based exacerbations are also associated with direct healthcare costs in the form of additional medications and physician visits or hospitalisations, and therefore this definition is useful for economic analyses [25]. Disadvantages include that event-based definitions can be confounded by patient socioeconomic status, geography and access to healthcare resources. By definition, to achieve an event a patient has to make contact with a healthcare professional and the healthcare professional has to decide the patient warrants treatment. Those with limited access to healthcare resources, whether because of geographic isolation, immobility or poverty, will necessarily experience fewer recorded events [26].

Studies that have assessed the incidence of symptom-based AECOPDs compared to event-based AECOPDs in the same patients followed over time suggest that observed exacerbation rates are much higher if symptom-based definitions are used. The Investigating New Standards for Prophylaxis in Reducing Exacerbations (INSPIRE) study compared the incidence of AECOPD using symptom-based definitions and a treatment-based definition and found that the incidence rate was three AECOPDs per patient-year if a symptom-based definition was used and 1.5 AECOPDs per patient-year if an event-based definition was used, suggesting that $50 \%$ of symptom-defined COPD exacerbations are not treated by physicians [27].

\section{Pitfalls and challenges}

Despite the best efforts of clinicians and guideline committees, current definitions of COPD exacerbation are imperfect and fraught with problems. The cardinal symptoms of a COPD exacerbation are nonspecific and can represent many disorders, including but not limited to pneumonia, congestive heart failure, acute coronary syndrome and pulmonary embolism (table 1) [17]. Bacterial or viral infection can precede an

TABLE 1 Causes of exacerbations or exacerbation-like symptoms in chronic obstructive pulmonary disease

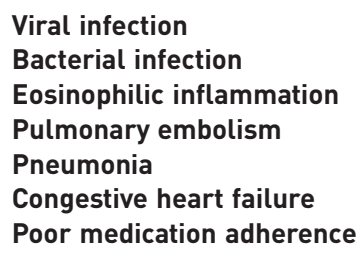


exacerbation, but in a significant proportion of events the inciting factor is unclear [28, 29]. Additionally, exacerbations may not necessarily be limited to COPD; recent literature suggests that those at risk for COPD (i.e. those with a significant smoking history) but without airflow obstruction may experience a similar number of exacerbation-like events compared to those with airflow obstruction [30-33]. Moreover, many exacerbations are not reported to physicians, making the true prevalence uncertain $[1,34]$.

The foundation of the definition of an exacerbation is the patient's perception of an increase in symptoms. Increased dyspnoea has been shown in several studies to be a risk factor for exacerbations and mortality, alone and as part of a multidimensional index [35-37]. However, it is possible that the perception of respiratory symptoms varies among individuals. A recent study found that those who experienced frequent exacerbations had heightened dyspnoea perception during $\mathrm{CO}_{2}$ rebreathing compared to those with infrequent exacerbations [38]. How this relates to the development of exacerbations remains uncertain, and may lead to more questions than answers. For instance, it is unclear if airway inflammation is greater in those patients with heightened dyspnoea perception and if this explains the link between dyspnoea perception and elevated risk of exacerbation [29, 39].

The necessity of determining the aetiology of an exacerbation remains controversial. It has been estimated that viral and bacterial infection are responsible for the majority of COPD exacerbations (table 1). Although viral infection can be detected using rapid PCR-based tests [40], revealing a viral aetiology may not result in a change in management outside of the identification of influenza. Additionally, the detection of bacteria in a patient with a COPD exacerbation does not necessarily distinguish a colonising organism from a pathogen, nor does it distinguish a lower respiratory tract infection from exacerbation. As such, current guidelines do not recommend obtaining sputum cultures because they are not always feasible or reliable, particularly in the outpatient setting [16]. Moreover, there are conflicting data on whether antibiotics are useful in exacerbations even in the presence of sputum purulence [41-43]. Newer technologies such as the electronic nose may help distinguish COPD exacerbations with concurrent infection from those without infection [44]. Tools such as these are greatly needed to help guide the use of antibiotics during exacerbations.

Phenotyping of exacerbations remains imperfect; our current classification scheme does not adequately capture their diversity or complexity. This complexity is partially addressed by the division of COPD exacerbations into mild, moderate and severe categories, which are conventionally described as requiring increased short-acting bronchodilator use, treatment with antibiotics and/or steroids, or an emergency room visit or hospitalisation, respectively. The GOLD committee also recommended that severe exacerbations be subclassified into no respiratory failure, acute respiratory failure - non-life-threatening, and acute respiratory failure - life-threatening [16]. However, questions regarding this classification remain. For example, how do we characterise mild exacerbations that only require an increased use of bronchodilators? Are they true exacerbations or do they represent a slightly exaggerated increase in day-to-day symptom variability? What causes these minor increases in symptoms? How do we categorise exacerbations that require treatment with antibiotics alone? Could these exacerbations be lower respiratory tract infections and not necessarily exacerbations of underlying COPD? Finally, none of these descriptors of COPD exacerbations specify an underlying pathophysiology.

One of the rate-limiting steps in diagnosing an exacerbation is ensuring that a physician is made aware of them. Unfortunately, many exacerbations are not reported. In one study of 128 COPD patients followed for 6 years, 1099 exacerbations identified by daily symptom diaries were recorded; however, 441 of these diary card exacerbations (40.2\%) were not reported to a physician [45]. Another study of 61 patients found similar results [1].

Additionally, recall of exacerbations is poor. In the Subpopulations and Intermediate Outcome Measures in COPD Study (SPIROMICS), 68 subjects with and without COPD had medical questionnaires taken at baseline and then again 6 weeks later to determine whether they had experienced AECOPDs in the previous year. There was a disparity in patient responses at baseline and 6 weeks later in 20 of 68 subjects (29\%). Six patients reported more exacerbations experienced within the previous year at 6 weeks compared with at baseline, and 14 reported fewer exacerbations [46]. This suggests that our definition is not specific enough for patients to identify exacerbations, and/or that patient recall of exacerbation events is faulty.

Several studies of the "frequent exacerbator" phenotype have been published recently. Although the frequent exacerbator may be responsible for a significant proportion of healthcare utilisation in COPD, the true prevalence of this phenotype is not clear. In the Evaluation of COPD Longitudinally to Identify Predictive Surrogate Endpoints (ECLIPSE) study, a history of previous exacerbations was the greatest risk factor for future exacerbations [8]. However, of those with two or more exacerbations in the year prior to enrolment, only $58 \%$ had frequent exacerbations in the first year, and only $60 \%$ of those with frequent exacerbations in the first year had frequent exacerbations in the second year. Therefore, $40-42 \%$ of the 
subjects had fewer than the anticipated number of exacerbations in the following year. A secondary analysis of the ECLIPSE study data determined that lower lung function was associated with a switch from an infrequent to a frequent exacerbator type, but that no parameter clearly predicted a change in exacerbation frequency category [47]. In a similar observational study, 32.2\% of 59 subjects with mild exacerbations during the first year experienced exacerbations in the second year [48]. Similarly, $40 \%$ of 60 patients with moderate or severe exacerbations during the first year had the same severity of exacerbation during the second year. Thus, it seems that the frequent exacerbator phenotype can vary year to year and does not necessarily convey a strong positive predictive value for future events.

These findings come with caveats. The definition of a frequent exacerbator has varied in prior literature, making it difficult to accurately summarise the data. Some studies have used a threshold of three or more exacerbations per year [1,49,50], whereas others have used a threshold as low as $\geqslant 1.5$ per year [2]. Additionally, recent literature has revealed that this phenotype is not as common as once believed. In the SPIROMICS cohort, only 82 of 1105 subjects (7\%) had at least one exacerbation per year during 3 years of follow-up, and only 23 (2\%) had two or more exacerbations per year [51].

Another topic that deserves further attention is determining when exacerbations begin and end. Using home diary card data, an exacerbation has been defined as the first of two or more consecutive days on which the patient records two or more new or worsening respiratory symptoms [34]. AARON et al. [52] used this definition and showed that in 1115 of 1995 COPD exacerbations (56\%) recorded using home diary cards, the onset of exacerbation was sudden and the exacerbation threshold was crossed on the same day that respiratory symptoms began. In contrast, $44 \%$ of exacerbations were characterised by gradual onset of symptoms. Patients who experienced sudden onset exacerbations had greater mean daily symptom scores, greater peak symptom scores and shorter median recovery times back to baseline health status (11 versus 13 days, $\mathrm{p}<0.001)$ [52].

The hallmark of the determination of exacerbation recovery is the return of symptoms back to baseline values. A study by WiLKINSON et al. [45] defined the recovery of an exacerbation as a return of respiratory symptoms to baseline levels for a period of 3 days. In this study, median time to recovery was 10.7 days. A more recent study examining the utility of the EXACT found that recovery of exacerbations was approximately 12 days [53]. However, an older study by SEEMUNGAL et al. [34] found that only $75.2 \%$ of 101 patients with moderate to severe COPD had "recovery" by 35 days as defined by their peak expiratory flow, and $7.1 \%$ of them had permanent reductions in peak expiratory flow at 91 days. Therefore, perhaps a newer paradigm for determining exacerbation recovery is needed. Newer technologies, e.g. activity and heart rate monitors, portable continuous pulse oximeters and telemedicine, could potentially be used as tools not only to help identify the symptoms and signs that herald an impending exacerbation but also to aid in determining exacerbation recovery. Thus far, current literature has focused on the use of activity monitors to detect daily physical activity in COPD but not in the detection of a new exacerbation or recovery from one [54-56].

\section{Opportunities for improvement}

Current definitions of COPD exacerbations are limited by the subjective nature of the symptoms used to define their occurrence, the nonspecificity of symptoms for pulmonary versus cardiac origin, patient non-reporting of exacerbation events to their healthcare providers and the variable response of clinicians to patients' reports of symptoms. The lack of an objective biomarker indicating the onset of the event or its severity and prognosis is a major problem with the current definition of exacerbation. A new, easy to use and objective definition of exacerbation is needed that incorporates symptom change with biomarker characterisation. An example from another area of medicine would be the definition of acute coronary syndromes. These are diagnosed based on a patient's history of angina-type chest pain, with the use of specific diagnostic biomarkers, including characteristic findings on ECG and changes in serum cardiac troponin, to help confirm the diagnosis and further phenotype the syndrome (myocardial infarction versus unstable angina). If symptoms of AECOPD could be combined with a sensitive and specific biomarker for AECOPD, then diagnosing AECOPD would become less subjective and hence more reliable.

\section{Biomarkers for AECOPD}

There is currently no ideal biomarker for identifying AECOPD. Acute changes in lung function (forced expiratory volume in $1 \mathrm{~s}\left(\mathrm{FEV}_{1}\right)$ ) or the $\mathrm{FEV}_{1}$ /forced vital capacity ratio are not sensitive, and do not correlate well with AECOPD [57, 58]. Commonly measured serum biomarkers such as C-reactive protein (CRP) or fibrinogen are nonspecific when used independently and are elevated in many other acute or chronic inflammatory or infectious states $[39,59]$.

Several plasma biomarkers of airway infection and inflammation have been studied in COPD exacerbations, but none have been found to be suitable for clinical use. A recent study of 86 Spanish 
patients hospitalised with AECOPD used multilevel network analysis to investigate pathobiological mechanisms of exacerbations and tried to identify biomarkers that could improve the specificity of the diagnosis. The investigators found that exacerbations were characterised by a disruption of the structure of the correlation network observed during convalescence, indicating less resilience and homeokinesis during exacerbations. A trio of biomarkers characterised by dyspnoea levels $\geqslant 5$ (on a visual analogue scale that ranges from 0 to 10$), \mathrm{CRP} \geqslant 3 \mathrm{mg} \cdot \mathrm{L}^{-1}$ and $\geqslant 70 \%$ circulating neutrophils had a specificity of 0.96 , a sensitivity of 0.90 , negative predictive value of 0.88 and positive predictive value of 0.97 for the identification of AECOPD [60].

Previous studies have identified four potential phenotypes of COPD exacerbations: bacterial, viral, eosinophilic and pauci-inflammatory [61]. To date, highly sensitive and specific biomarkers to accurately phenotype AECOPD have proved elusive. Biomarkers associated with bacteria-driven exacerbations have poor specificity: a sputum interleukin-1 $\beta$ level of $130 \mathrm{pg} \cdot \mathrm{mL}^{-1}$ had a sensitivity and specificity of $80 \%$ and $60 \%$ respectively, and a serum CRP level of $10 \mathrm{mg} \cdot \mathrm{L}^{-1}$ had a sensitivity and specificity of only $65 \%$. Similarly, a serum eosinophil count $\geqslant 2 \%$ had a sensitivity and specificity of $90 \%$ and $60 \%$ for identifying eosinophilic-associated exacerbations [62]. At this point, accurate phenotyping of AECOPD has not proven feasible in routine clinical practice.

Recent attention has turned to using alternative biomarkers to phenotype AECOPD. Procalcitonin, the prohormone of calcitonin, is released in different tissues in response to bacterial, but not viral, infections or nonspecific inflammation [63]. For this reason, procalcitonin can theoretically be used as a biomarker to help differentiate between COPD exacerbations caused by bacteria and exacerbations due to other aetiologies. A recent meta-analysis of eight trials evaluating 1062 patients with AECOPD demonstrated that procalcitonin-based protocols could decrease antibiotic prescription for AECOPD (relative risk 0.56, 95\% CI 0.43-0.73) without adversely affecting clinical outcomes such as rate of treatment failure, length of hospitalisation, exacerbation recurrence rate or mortality [64]. In contrast, a trial of 1656 patients who presented to the emergency department with a suspected lower respiratory tract infection found that the provision of procalcitonin assay results, along with instructions on their interpretation, to emergency department and hospital-based clinicians did not result in less use of antibiotics than did usual care among patients with suspected lower respiratory tract infections [65]. To date, it is not known whether procalcitonin-based protocols are cost effective and change clinical outcomes for COPD in routine clinical practice.

\section{A potential new definition for COPD exacerbations}

Table 2 shows a proposed new definition for COPD exacerbation as well as a grading system to characterise COPD clinical states. This new definition and the grading system have been adapted and refined from a "precision medicine" proposal advanced by AGusti et al. [66] and from a paper by CelLi et al. [67] describing grading COPD clinical states. Newer evidence generated by BAFADHEL et al. [61] and Noell et al. [60] has been incorporated into the definition. According to the new proposed definition, the clinical hallmarks of COPD exacerbation are increased dyspnoea $(\geqslant 5$ on a visual analogue scale that ranges from 0 to 10), sometimes but not necessarily accompanied by increased cough, sputum volume or

TABLE 2 Potential new exacerbation definition (analogous to events in coronary artery disease)

Coronary artery disease

COPD

Myocardial infarction (heart attack)
Chest pain
and
Abnormal ECG
and
Elevated troponin

\section{Cardiogenic shock}

Same syndrome, plus shock

\section{COPD exacerbation (lung attack)}

Increased dyspnoea ( $\geqslant 5$ on a visual analogue scale that ranges from 0 to 10 ) and

$\mathrm{O}_{2}$ desaturation $\leqslant 4 \%$ below that of stable state, or $\mathrm{O}_{2}$ saturation $\leqslant 90 \%$ if no baseline value available and $\mathrm{CRP} \geqslant 3 \mathrm{mg} \cdot \mathrm{L}^{-1}$ and

Circulating blood neutrophils $\geqslant 9000$ cells $\cdot \mathrm{mm}^{-3}$ or circulating blood eosinophils $\geqslant 2 \%$ and

No pneumonia or congestive heart failure or pneumothorax or pleural effusion on chest radiography Respiratory failure

Same syndrome, plus $\mathrm{PaCO}_{2}>45 \mathrm{mmHg}$

Adapted from references $[65,66]$. COPD: chronic obstructive pulmonary disease; CRP: C-reactive protein; $\mathrm{PaCO}_{2}$ : arterial carbon dioxide tension. 
sputum purulence. Laboratory evidence necessary to confirm a COPD exacerbation includes oxygen desaturation $\leqslant 4 \%$ below that of stable state, elevated CRP $\left(\geqslant 3 \mathrm{mg} \cdot \mathrm{L}^{-1}\right)$ and circulating blood neutrophilia $\left(\geqslant 9000\right.$ neutrophils $\left.\cdot \mathrm{mm}^{-3}\right)$ or circulating blood eosinophilia $(\geqslant 2 \%$ blood eosinophils). Other aetiologies of respiratory decompensation should be ruled out by obtaining chest radiography that excludes pneumonia, congestive heart failure and pleural effusion/pneumothorax. When the same exacerbation syndrome is accompanied by hypercapnia and acute respiratory acidosis, the syndrome can be further defined as "exacerbation with respiratory failure".

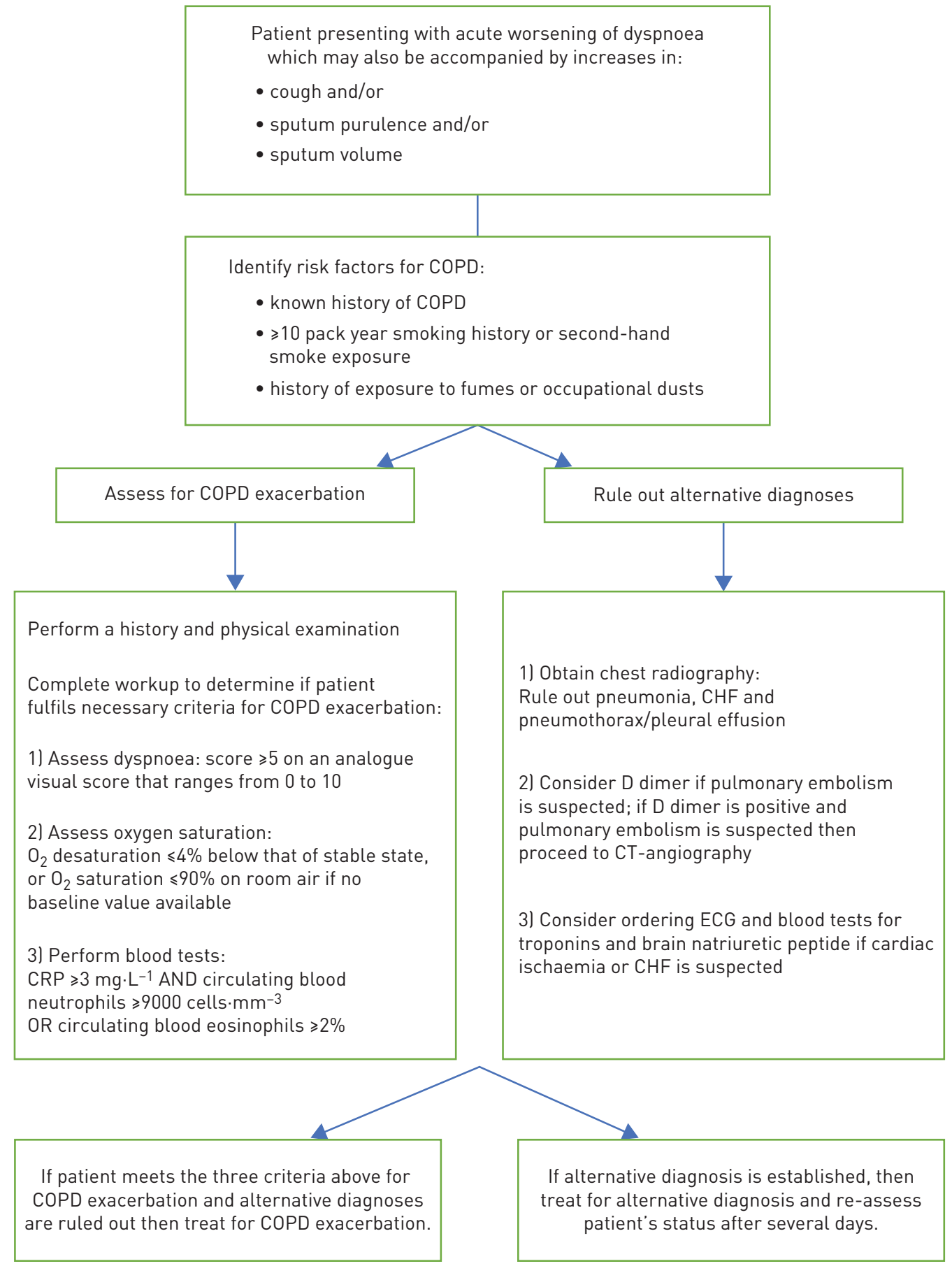

FIGURE 1 A suggested clinical approach to suspected chronic obstructive pulmonary disease (COPD) exacerbations. Confirmation of COPD exacerbation requires symptoms compatible with COPD exacerbation; a patient history of COPD or risk factors for COPD; laboratory investigations compatible with COPD exacerbation; and laboratory investigations that rule out other diagnoses. CRP: C-reactive protein; CHF: congestive heart failure; CT: computed tomography. 
Figure 1 depicts a flow diagram describing a suggested clinical approach to suspected COPD exacerbations. The investigations needed to confirm a diagnosis of COPD exacerbation, as well as investigations needed to rule out alternative diagnoses, are indicated.

Although our proposed definition for COPD exacerbation appears to be more specific than that which currently exists, this proposed definition for AECOPD and our proposed approach for diagnosing AECOPD need to be validated in prospective clinical studies.

\section{Conclusions}

A more accurate definition of COPD exacerbation is critical for obtaining a better understanding of diagnosis and treatment as well as the true prevalence of COPD exacerbations. Currently employed definitions of AECOPD are imperfect and nonspecific. A wide variety of medical conditions, e.g. congestive heart failure, anaemia and pulmonary embolism, can cause increased respiratory symptoms and dyspnoea in a patient with COPD. Any of these conditions can potentially and mistakenly meet current definitions of "COPD exacerbation". In the absence of a single biomarker to easily identify a COPD exacerbation in clinical practice, it seems likely that an improved definition of AECOPD, such as the new proposed definition provided within this review, will incorporate a combination of 1) clinical symptoms, such as increased dyspnoea, cough or sputum production; 2) positive laboratory biomarkers suggesting AECOPD, such as elevated serum CRP and serum neutrophilia or eosinophilia; and 3) exclusion of other potential aetiologies via negative results on chest radiography and negative laboratory test results for cardiac, pulmonary or haematological disorders that can mimic AECOPD. Future research will hopefully elucidate better biomarkers that are both sensitive and highly specific for AECOPD and will ultimately lead to greater diagnostic accuracy for these potentially devastating events that shape the natural history of COPD.

Conflict of interest: None declared.

\section{References}

1 Seemungal TA, Donaldson GC, Paul EA, et al. Effect of exacerbation on quality of life in patients with chronic obstructive pulmonary disease. Am J Respir Crit Care Med 1998; 157: 1418-1422.

2 Kanner RE, Anthonisen NR, Connett JE, et al. Lower respiratory illnesses promote FEV1 decline in current smokers but not ex-smokers with mild chronic obstructive pulmonary disease: results from the lung health study. Am J Respir Crit Care Med 2001; 164: 358-364.

3 Donaldson GC, Seemungal TA, Bhowmik A, et al. Relationship between exacerbation frequency and lung function decline in chronic obstructive pulmonary disease. Thorax 2002; 57: 847-852.

4 Aaron SD, Vandemheen KL, Clinch JJ, et al. Measurement of short-term changes in dyspnea and disease-specific quality of life following an acute COPD exacerbation. Chest 2002; 121: 688-696.

5 Groenewegen KH, Schols AM, Wouters EF. Mortality and mortality-related factors after hospitalization for acute exacerbation of COPD. Chest 2003; 124: 459-467.

6 Makris D, Moschandreas J, Damianaki A, et al. Exacerbations and lung function decline in COPD: new insights in current and ex-smokers. Respir Med 2007; 101: 1305-1312.

7 Dransfield MT, Kunisaki KM, Strand MJ, et al. Acute exacerbations and lung function loss in smokers with and without chronic obstructive pulmonary disease. Am J Respir Crit Care Med 2017; 195: 324-330.

8 Hurst JR, Vestbo J, Anzueto A, et al. Evaluation of COPD Longitudinally to Identify Predictive Surrogate Endpoints (ECLIPSE) Investigators. Susceptibility to exacerbation in chronic obstructive pulmonary disease. N Engl J Med 2010; 363: 1128-1138.

9 Han MK, Kazerooni EA, Lynch DA, et al. Chronic obstructive pulmonary disease exacerbations in the COPDGene study: associated radiologic phenotypes. Radiology 2011; 261: 274-282.

10 Kim V, Davey A, Comellas AP, et al. Clinical and computed tomographic predictors of chronic bronchitis in COPD: a cross sectional analysis of the COPDGene study. Respir Res 2014; 15: 52.

11 Aaron SD, Fergusson D, Marks GB, et al. Counting, analysing and reporting exacerbations of COPD in randomised controlled trials. Thorax 2008; 63: 122-128.

12 Suissa S, Ernst P, Vandemheen KL, et al. Methodological issues in therapeutic trials of COPD. Eur Respir J 2008; 31: 927-933.

13 Anthonisen NR, Manfreda J, Warren CP, et al. Antibiotic therapy in exacerbations of chronic obstructive pulmonary disease. Ann Intern Med 1987; 106: 196-204.

14 Paggiaro PL, Dahle R, Bakran I, et al. Multicentre randomised placebo-controlled trial of inhaled fluticasone propionate in patients with chronic obstructive pulmonary disease. International COPD Study Group. Lancet 1998; 351: 773-780.

15 Rodriguez-Roisin R. Toward a consensus definition for COPD exacerbations. Chest 2000; 117: 398S-401S.

16 Vogelmeier CF, Criner GJ, Martinez FJ, et al. Global Strategy for the Diagnosis, Management, and Prevention of Chronic Obstructive Lung Disease 2017 Report: GOLD Executive Summary. Eur Respir J 2017; 49: 1750214.

17 Beghe B, Verduri A, Roca M, et al. Exacerbation of respiratory symptoms in COPD patients may not be exacerbations of COPD. Eur Respir J 2013; 41: 993-995.

18 Quint JK, Donaldson GC, Hurst JR, et al. Predictive accuracy of patient-reported exacerbation frequency in COPD. Eur Respir J 2011; 37: 501-507.

19 Leidy NK, Murray LT. Patient-reported outcome (PRO) measures for clinical trials of COPD: the EXACT and E-RS. COPD 2013; 10: 393-398. 
20 Langsetmo L, Platt RW, Ernst P, et al. Underreporting exacerbation of chronic obstructive pulmonary disease in a longitudinal cohort. Am J Respir Crit Care Med 2008; 177: 396-401.

21 Vijayasaratha K, Stockley RA. Reported and unreported exacerbations of COPD: analysis by diary cards. Chest 2008; 133: 34-41.

22 Wolpin S, Nguyen HQ, Donesky-Cuenco D, et al. Effects of automated prompts for logging symptom and exercise data on mobile devices in patients with chronic obstructive pulmonary disease. Comput Inform Nurs 2011; 29: TC3-TC8.

23 Singh D, Kampschulte J, Wedzicha JA, et al. A trial of beclomethasone/formoterol in COPD using EXACT-PRO to measure exacerbations. Eur Respir J 2013; 41: 12-17.

24 Mackay AJ, Donaldson GC, Patel AR, et al. Detection and severity grading of COPD exacerbations using the exacerbations of chronic pulmonary disease tool (EXACT). Eur Respir J 2014; 43: 735-744.

25 Najafzadeh M, Marra CA, Sadatsafavi M, et al. Cost effectiveness of therapy with combinations of long acting bronchodilators and inhaled steroids for treatment of COPD. Thorax 2008; 63: 962-967.

26 Hunter LC, Lee RJ, Butcher I, et al. Patient characteristics associated with risk of first hospital admission and readmission for acute exacerbation of chronic obstructive pulmonary disease (COPD) following primary care COPD diagnosis: a cohort study using linked electronic patient records. BMJ Open 2016; 6: e009121.

27 Wedzicha JA, Calverley PM, Seemungal TA, et al. The prevention of chronic obstructive pulmonary disease exacerbations by salmeterol/fluticasone propionate or tiotropium bromide. Am J Respir Crit Care Med 2008; 177: 19-26.

28 Seemungal T, Harper-Owen R, Bhowmik A, et al. Respiratory viruses, symptoms, and inflammatory markers in acute exacerbations and stable chronic obstructive pulmonary disease. Am J Respir Crit Care Med 2001; 164: 1618-1623.

29 Sapey E, Stockley RA. COPD exacerbations. 2: aetiology. Thorax 2006; 61: 250-258.

30 Bowler RP, Kim V, Regan E, et al. Prediction of acute respiratory disease in current and former smokers with and without COPD. Chest 2014; 146: 941-950.

31 Tan WC, Bourbeau J, Hernandez P, et al. Exacerbation-like respiratory symptoms in individuals without chronic obstructive pulmonary disease: results from a population-based study. Thorax 2014; 69: 709-717.

32 Martinez CH, Kim V, Chen Y, et al. The clinical impact of non-obstructive chronic bronchitis in current and former smokers. Respir Med 2014; 108: 491-499.

33 Woodruff PG, Barr RG, Bleecker E, et al. Clinical significance of symptoms in smokers with preserved pulmonary function. $N$ Engl J Med 2016; 374: 1811-1821.

34 Seemungal TA, Donaldson GC, Bhowmik A, et al. Time course and recovery of exacerbations in patients with chronic obstructive pulmonary disease. Am J Respir Crit Care Med 2000; 161: 1608-1613.

35 Nishimura K, Izumi T, Tsukino M, et al. Dyspnea is a better predictor of 5-year survival than airway obstruction in patients with COPD. Chest 2002; 121: 1434-1440.

36 Celli BR, Cote CG, Marin JM, et al. The body-mass index, airflow obstruction, dyspnea, and exercise capacity index in chronic obstructive pulmonary disease. N Engl J Med 2004; 350: 1005-1012.

37 Marin JM, Carrizo SJ, Casanova C, et al. Prediction of risk of COPD exacerbations by the BODE index. Respir Med 2009; 103: 373-378.

38 Scioscia G, Blanco I, Arismendi E, et al. Different dyspnoea perception in COPD patients with frequent and infrequent exacerbations. Thorax 2017; 72: 117-121.

39 Hurst JR, Perera WR, Wilkinson TM, et al. Systemic and upper and lower airway inflammation at exacerbation of chronic obstructive pulmonary disease. Am J Respir Crit Care Med 2006; 173: 71-78.

40 Caliendo AM. Multiplex PCR and emerging technologies for the detection of respiratory pathogens. Clin Infect Dis 2011; 52: Suppl. 4, S326-S330.

41 Vollenweider DJ, Jarrett H, Steurer-Stey CA, et al. Antibiotics for exacerbations of chronic obstructive pulmonary disease. Cochrane Database Syst Rev 2012; 12: CD010257.

42 Miravitlles M, Kruesmann F, Haverstock D, et al. Sputum colour and bacteria in chronic bronchitis exacerbations a pooled analysis. Eur Respir J 2012; 39: 1354-1360.

43 van Velzen P, Ter Riet G, Bresser P, et al. Doxycycline for outpatient-treated acute exacerbations of COPD: a randomised double-blind placebo-controlled trial. Lancet Respir Med 2017; 5: 492-499.

44 Shafiek H, Fiorentino F, Merino JL, et al. Using the electronic nose to identify airway infection during COPD exacerbations. PLoS One 2015; 10: e0135199.

45 Wilkinson TM, Donaldson GC, Hurst JR, et al. Early therapy improves outcomes of exacerbations of chronic obstructive pulmonary disease. Am J Respir Crit Care Med 2004; 169: 1298-1303.

46 Anderson WH, Ha JW, Couper DJ, et al. Variability in objective and subjective measures affects baseline values in studies of patients with COPD. PLoS One 2017; 12: e0184606.

47 Donaldson GC, Mullerova H, Locantore N, et al. Factors associated with change in exacerbation frequency in COPD. Respir Res 2013; 14: 79.

48 Sato M, Chubachi S, Sasaki M, et al. Impact of mild exacerbation on COPD symptoms in a Japanese cohort. Int J Chron Obstruct Pulmon Dis 2016; 11: 1269-1278.

49 Miravitlles M, Ferrer M, Pont A, et al. Effect of exacerbations on quality of life in patients with chronic obstructive pulmonary disease: a 2 year follow up study. Thorax 2004; 59: 387-395.

50 Soler JJ, Sanchez L, Roman P, et al. Risk factors of emergency care and admissions in COPD patients with high consumption of health resources. Respir Med 2004; 98: 318-329.

51 Han MK, Quibrera PM, Carretta EE, et al. Frequency of exacerbations in patients with chronic obstructive pulmonary disease: an analysis of the SPIROMICS cohort. Lancet Respir Med 2017; 5: 619-626.

52 Aaron SD, Donaldson GC, Whitmore GA, et al. Time course and pattern of COPD exacerbation onset. Thorax 2012; 67: 238-243.

53 Leidy NK, Wilcox TK, Jones PW, et al. Standardizing measurement of chronic obstructive pulmonary disease exacerbations. Reliability and validity of a patient-reported diary. Am J Respir Crit Care Med 2011; 183: 323-329.

54 Benzo R. Activity monitoring in chronic obstructive pulmonary disease. J Cardiopulm Rehabil Prev 2009; 29: 341-347. 
55 Cohen MD, Cutaia M. A novel approach to measuring activity in chronic obstructive pulmonary disease: using 2 activity monitors to classify daily activity. J Cardiopulm Rehabil Prev 2010; 30: 186-194.

56 Ehsan $\mathrm{M}$, Khan $\mathrm{R}$, Wakefield $\mathrm{D}$, et al. A longitudinal study evaluating the effect of exacerbations on physical activity in patients with chronic obstructive pulmonary disease. Ann Am Thorac Soc 2013; 10: 559-564.

57 Stevenson NJ, Walker PP, Costello RW, et al. Lung mechanics and dyspnea during exacerbations of chronic obstructive pulmonary disease. Am J Respir Crit Care Med 2005; 172: 1510-1516.

58 Parker CM, Voduc N, Aaron SD, et al. Physiological changes during symptom recovery from moderate exacerbations of COPD. Eur Respir J 2005; 26: 420-428.

59 Hurst JR, Donaldson GC, Perera WR, et al. Use of plasma biomarkers at exacerbation of chronic obstructive pulmonary disease. Am J Respir Crit Care Med 2006; 174: 867-874.

60 Noell G, Cosio BG, Faner R, et al. Multi-level differential network analysis of COPD exacerbations. Eur Respir J 2017; 50; 1700075 .

61 Bafadhel M, McKenna S, Terry S, et al. Acute exacerbations of chronic obstructive pulmonary disease: identification of biologic clusters and their biomarkers. Am J Respir Crit Care Med 2011; 184: 662-671.

62 Bafadhel M, McKenna S, Terry S, et al. Blood eosinophils to direct corticosteroid treatment of exacerbations of chronic obstructive pulmonary disease: a randomized placebo-controlled trial. Am J Respir Crit Care Med 2012; 186: 48-55.

63 Stolz D, Christ-Crain M, Bingisser R, et al. Antibiotic treatment of exacerbations of COPD: a randomized, controlled trial comparing procalcitonin-guidance with standard therapy. Chest 2007; 131: 9-19.

64 Mathioudakis AG, Chatzimavridou-Grigoriadou V, Corlateanu A, et al. Procalcitonin to guide antibiotic administration in COPD exacerbations: a meta-analysis. Eur Respir Rev 2017; 26; 160073.

65 Huang DT, Yealy DM, Filbin MR, et al. Procalcitonin-guided use of antibiotics for lower respiratory tract infection. N Engl J Med 2018; 379: 236-249.

66 Agusti A, Faner R, Celli B, et al. Precision medicine in COPD exacerbations. Lancet Respir Med 2018; 6: 657-659.

67 Celli BR, Barnes PJ. Exacerbations of chronic obstructive pulmonary disease. Eur Respir J 2007; 29: 1224-1238. 